\title{
Knockdown of microRNA-130b improves doxorubicin sensitivity in bladder urothelial carcinoma by negatively regulating cylindromatosis expression
}

Bo Li, Hui Zhang

China Medical University, Shenyang, China

Submitted: 15 March 2018; Accepted: 25 July 2018

Online publication: 17 July 2019

Arch Med Sci 2021; 17 (4): 1038-1043

DOI: https://doi.org/10.5114/aoms.2019.86622

Copyright @ 2019 Termedia \& Banach

\begin{abstract}
Introduction: Chemotherapeutic resistance reduces the sensitivity of bladder urothelial carcinoma (BUC) to chemotherapeutic drugs and contributes a barrier leading to treatment failure. The purpose of this research project is to investigate the regulatory effects of miR-130b on chemotherapeutic drug resistance of BUC and its mechanism.

Material and methods: The relative expression of miRNA-130b and cylindromatosis (CYLD) was examined using real-time quantitative PCR. The cell proliferation and doxorubicin sensitivity were detected with the enhanced CCK-8 assay. The specific combination of miR-130b and CYLD was verified with the luciferase reporter gene assay. Protein expression was detected by Western blot.

Results: Our study found that miR-130b was up-regulated in doxorubicin-insensitive BUC tissues and cell lines, and its high expression was negatively related to doxorubicin sensitivity in BUC. The miR-130b knockdown reduced the $\mathrm{IC}_{50}$ of doxorubicin and improved doxorubicin sensitivity of J82/Dox and T24/Dox cells. For the regulation mechanism analysis of miR-130b, bioinformatics analysis software was used to predict the potential targets of miR-130b, including the CYLD gene. The following luciferase activities assay, quantitative real time-PCR and western blot identified the CYLD gene as a target of miR-130b. Knockdown of CYLD reversed miR-130b's regulatory roles in doxorubicin sensitivity in J82/Dox and T24/Dox cells.

Conclusions: High expression of miR-130b is negatively related to doxorubicin sensitivity in BUC, and knockdown of miR-130b improves doxorubicin sensitivity in BUC by negatively regulating CYLD expression. Our findings will provide guidance for the clinical chemotherapy of BUC.
\end{abstract}

Key words: antineoplastic agents, doxorubicin, microRNAs, urinary bladder neoplasms.

\section{Introduction}

Bladder cancer is a common malignant tumor in the urinary system. Bladder urothelial carcinomas (BUC) is the most representative histological classification of bladder cancer, which was called bladder transitional cell carcinoma (BTCC) before [1]. Surgical resection is the first choice for the treatment of BUC. Chemical therapy is also an important supplement to surgical treatment, which can significantly improve the prognosis of patients. Chemotherapy resistance severely limits the therapeutic effect and clinical application of chemotherapy [2]. Therefore, it is very import-

\author{
Corresponding author: \\ Dr. Hui Zhang \\ China Medical University \\ 36 Sanhao St \\ Heping Area \\ 110004 Shenyang, China \\ Phone: +18940257926 \\ E-mail: 839672449@qq.com
}


ant to study the mechanism of chemotherapy resistance in BUC and to find new therapeutic targets.

MicroRNA is a class of small molecule RNA that does not have the ability to encode protein. MicroRNA has complex physiological functions and participates in the genesis of a variety of complex diseases, including cancers [3-5]. MiR-130b was up-regulated and acted as an oncogene in some kinds of cancers [6-11]. Recent studies found that miR-130b was highly expressed in BUC and could promote cell migration and invasion in BUC $[9,12]$, but the biological function and molecular mechanism of miR-130b in chemotherapy resistance of BUC was still unknown. Bioinformatics predicted that the cylindromatosis (CYLD) gene might be a target of miR-130b. CYLD was a deubiquitinating enzyme located at 16q12.1 [13], and its low expression has been detected in many kinds of cancers [14-16].

This study will research the regulatory effects of miR-130b on chemotherapeutic drug resistance of BUC and its mechanism, and the consequences will contribute to BUC clinical chemotherapy.

\section{Material and methods}

\section{Clinical specimens}

A total of 71 BUC patients were treated in the Department of Urinary Surgery of Shengjing Hospital between November 2011 and February 2013. The BUC specimens were gathered using a cystoscope. After pathological diagnosis, doxorubicin chemotherapy was applied to all patients, and RECIST Response Evaluation Criteria were used to evaluate the treatment effects. All patients were divided into insensitive and sensitive groups; the former $(n=44)$ was deteriorated or stable, and the latter $(n=27)$ was partially or completely relieved. This study has been carried out with the approval of the Ethics Committees of Shengjing Hospital. All patients signed an informed consent form.

\section{Cell culture}

A human normal bladder epithelial cell line (HCV29) and human bladder urothelial carcinoma cell lines (J82 and T24) were obtained from the National Infrastructure of Cell Line Resource (China), and cultured in DMEM medium which included $10 \%$ fetal bovine serum (Cyagen, China) at the condition of $37^{\circ} \mathrm{C}$ and $5 \% \mathrm{CO}_{2}$. An doxorubicin-resistant cell line was established from J82 and T24 cell lines previously and named J82/Dox and T24/ Dox cell lines [17].

\section{Real-time quantitative PCR}

Total RNA was extracted from BUC tissues and cells with Trizol reagent, and synthesized to cDNA with the TIANScript RT Kit (TIANGEN, China). The expression of miR-130b and CYLD was examined with the Talent qPCR PreMix Kit (TIANGEN, China) in accordance with the instructions. A relative quantitative method was used to quantify the expression of miR-130b and CYLD. U6 and GAPDH were used as endogenous controls.

\section{Transfection}

The agonist and antagonist of miR-130b (miR$130 \mathrm{~b}(+)$ and miR-130b(-)) and their negative controls (miR-NC(+) and miR-NC(-)) were designed and synthesized by GenePharma Company (China). The silence vector of CYLD (sh-CYLD) and its negative control (sh-NC) were also designed and synthesized by GenePharma Company. All vectors and microRNAs were transfected using Lipofectamine 2000 (Thermo Fisher Scientific, USA) in accordance with instructions.

\section{Drug sensitivity assay}

Three thousand cells (T24 or T24/Dox) were placed in a well of 96-well plates. After $24 \mathrm{~h}$, doxorubicin $(0.1 \mu \mathrm{g} / \mathrm{ml}, 0.5 \mu \mathrm{g} / \mathrm{ml}, 1 \mu \mathrm{g} / \mathrm{ml}, 5 \mu \mathrm{g} / \mathrm{ml}$, $10 \mu \mathrm{g} / \mathrm{ml}$ ) was used to treat those cells $[17,18]$. The cell viability was examined using Enhanced Cell Counting Kit-8 (Beyotime, China) 48 h later; $10 \mu$ of enhanced CCK-8 solution was added to each well of a 96-well plate, and the plate was incubated for $2 \mathrm{~h}$ at $37^{\circ} \mathrm{C}$. The value of optical density at was detected with an MK3 microplate reader (Thermo Fisher Scientific, USA) at $450 \mathrm{~nm}$. Then, the data were used to calculate IC50 (half maximal inhibitory concentration).

\section{Luciferase reporter gene assay}

The TargetScan 7.0 software was selected to predict the target genes of miR-130b. The luciferase reporter vectors $p$-CYLD-mut (containing mutant binding site) and p-CYLD-wt (containing wildtype binding site) were designed and synthesized by GenePharma Company (China). MicroRNAs and constructed vectors were cotransfected to HEK 293T cells using Lipofectamine 2000 Reagent. Forty-eight hours later, the Dual Luciferase Reporter Gene Assay Kit (Beyotime, China) was applied to detect the luciferase activity in accordance with the instructions.

\section{Western blot}

Protein was extracted and the concentration determined with the BCA Protein Assay Kit (TIANGEN, China) in accordance with the instructions. Protein samples $(20 \mathrm{mg}$ ) were handled with SDSPAGE gel electrophoresis and transferred to PVDF membranes. The PVDF membrane was first hy- 

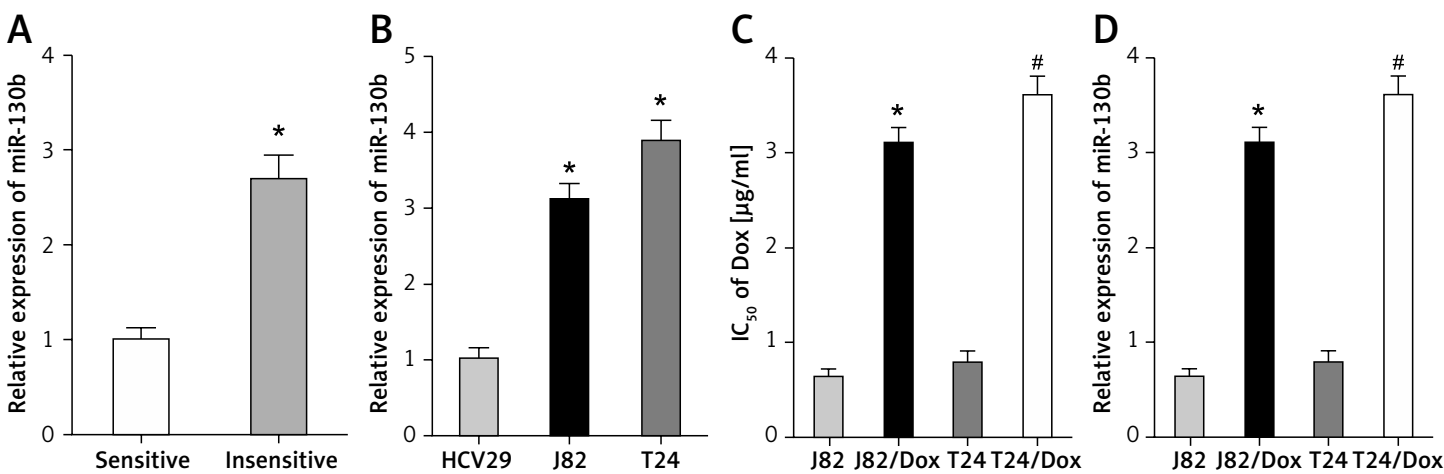
(27)

Figure 1. Over-expression of miR-130b is negatively correlated with doxorubicin sensitivity of BUC. A - The expression of miR-130b in doxorubicin-insensitive BUC patients was up-regulated compared with doxorubicin-sensitive BUC patients. ${ }^{*} p<0.05$ vs. sensitive group. B - The expression of miR-130b in 82 and T24 cells was higher than that in HCV29 cells. ${ }^{*} p<0.05$ vs. HCV29 cells. C - The IC50 of doxorubicin in J82/Dox and T24/Dox cells was higher than that in $\mathrm{J} 82$ and T24 cells. ${ }^{*} p<0.05$ vs. J82 cells, ${ }^{*} p<0.05$ vs. T24 cells. D - The miR-130b expression in J82/ Dox and T24/Dox cells increased compared with $J 82$ and T24 cells. ${ }^{*} p<0.05$ vs. J82 cells, ${ }^{*} p<0.05$ vs. T24 cells

bridized with CYLD antibody (\#4495, Cell Signaling, USA) and incubated with second antibody in turn. Then, the PVDF membrane was incubated with the Pro-light HRP Chemiluminescent Kit (TIANGEN, China). Then, the bands were detected and analyzed by ImageJ software (NIH, USA).

\section{Statistical analysis}

SPSS 22.0 (IBM, USA) was used for statistical analysis. All data were expressed as mean \pm standard deviation and analyzed with one-way ANOVA and Student's $t$-test. If the $p$-value was less than 0.05 , the difference was statistically significant.

\section{Results}

Over-expression of miR-130b is negatively correlated with doxorubicin sensitivity of BUC

In comparison with doxorubicin-sensitive BUC patients, the expression of miR-130b in doxoru-

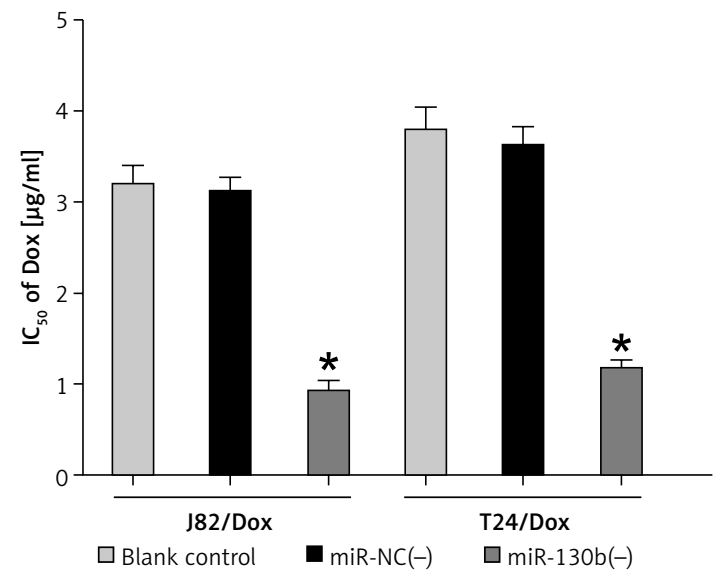

Figure 2. Knockdown of miR-130b reduced $I C_{50}$ of doxorubicin in doxorubicin-resistant cells. ${ }^{*} p<0.05$ vs. blank control or miR-NC(-) group bicin-insensitive BUC patients was up-regulated (Figure $1 \mathrm{~A}$ ), which provided preliminary evidence that miR-130b might be involved in the doxorubicin resistance of $B \cup C$.

To confirm this above finding, the expression of miR-130b was also examined in doxorubicin-sensitive and doxorubicin-resistant cell lines. The miR-130b expression in 182 and T24 cells was much higher than that in HCV29 cells (Figure $1 \mathrm{~B}$ ). J82/Dox and T24/Dox cells were resistant to doxorubicin, their IC50 to doxorubicin (3.11 \pm 0.16 $\mu \mathrm{g} / \mathrm{ml}$ and $3.61 \pm 0.21 \mu \mathrm{g} / \mathrm{ml}$ ) was higher than that in $J 82$ and T24 cells $(0.63 \pm 0.09 \mu \mathrm{g} / \mathrm{ml}$ and 3.61 $\pm 0.21 \mu \mathrm{g} / \mathrm{ml}$ ), and the resistant index (RI) was 4.94 and 4.57 respectively (Figure $1 \mathrm{C}$ ). Compared with 182 and T24 cells, the miR-130b expression in J82/Dox and T24/Dox cells increased obviously (Figure $1 \mathrm{D}$ ).

\section{Knockdown of miR-130b improved doxorubicin sensitivity of doxorubicin- resistant cells}

In J82/Dox and T24/Dox cells, the miR-130b expression was silenced by transfection with miR$130 \mathrm{~b}(-)$. MiR-130b knockdown reduced IC $\mathrm{I}_{50}$ of doxorubicin in J82/Dox and T24/Dox cells from $3.11 \pm 0.16$ $\mu \mathrm{g} / \mathrm{ml}$ and $3.61 \pm 0.21 \mu \mathrm{g} / \mathrm{ml}$ to $0.93 \pm 0.11 \mu \mathrm{g} / \mathrm{ml}$ and $1.17 \pm 0.08 \mu \mathrm{g} / \mathrm{ml}$ (Figure 2), which further revealed that miR-130b was involved in BUC doxorubicin resistance, and its knockdown could improve doxorubicin sensitivity of J82/Dox and T24/Dox cells.

\section{CYLD gene is a target of miR-130b in BUC cells}

As for the vital roles of miR-130b in BUC chemotherapy resistance, further mechanism analysis is necessary. The bioinformatics software TargetScan was selected to predict a binding site of miR-130b in the 190-196 bp of CYLD 3'-UTR (Figure 3 A). 
A

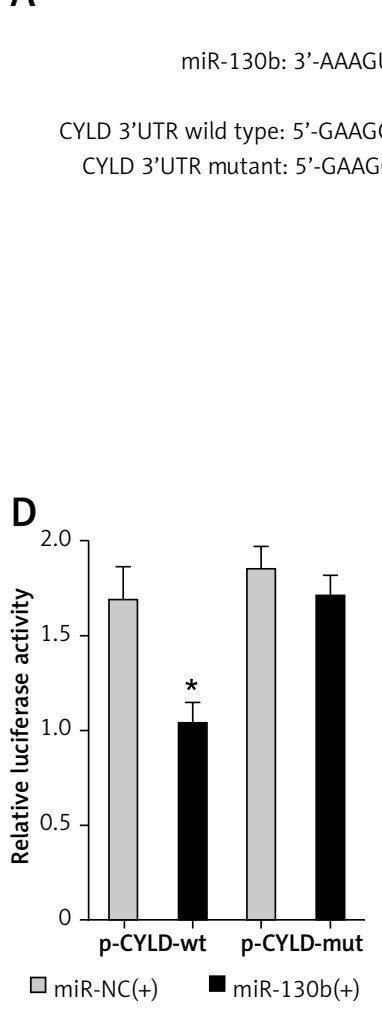

B

(190-196 BP)

AACGUGA C-5,

\|\|\|\|

CAUU UUGCACU C-3'

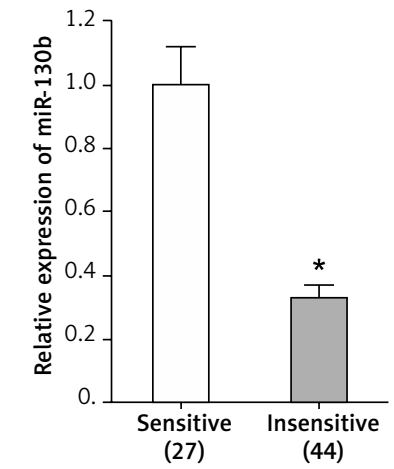

C

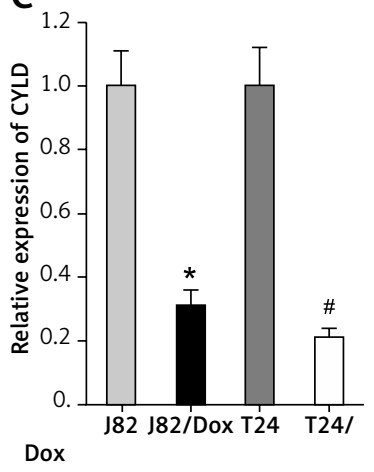

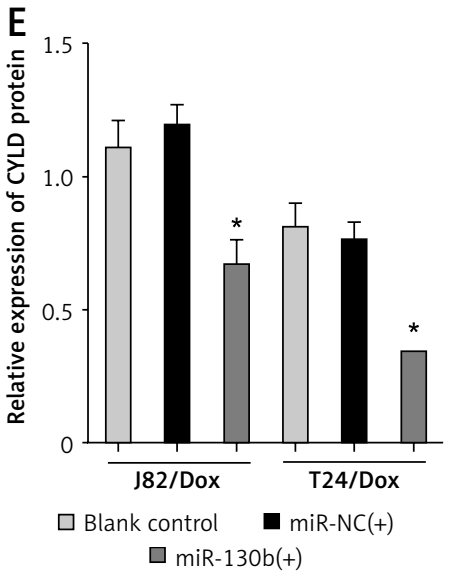

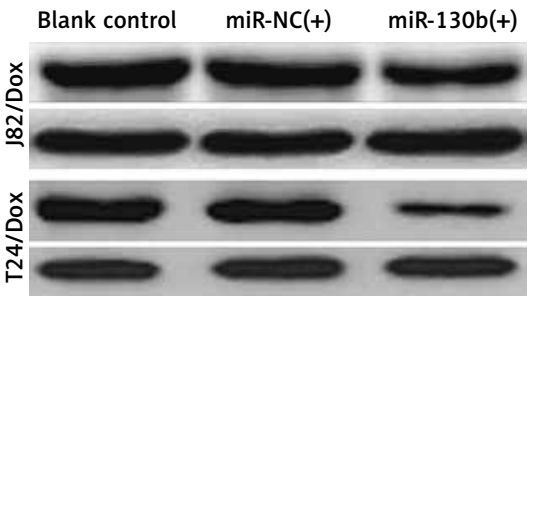

Figure 3. CYLD gene is a target of miR-130b in BUC cells. A - The binding site of miR-130b in the $3^{\prime} U T R$ of CYLD. B - The expression of CYLD in doxorubicin-insensitive BUC patients was down-regulated compared with doxorubicin-sensitive BUC patients. ${ }^{*} p<0.05$ vs sensitive group. C - The CYLD expression in J82/Dox and T24/Dox cells decreased compared with $J 82$ and T24 cells. ${ }^{*} p<0.05$ vs. $J 82$ cells, ${ }^{*} p<0.05$ vs. T24 cells. D - MiR-130b inhibited the relative luciferase activity by combining with wild-type binding site of CYLD. ${ }^{*} p<0.05$ vs. miR-NC(+) + p-CYLDwt. E - MiR-130b reduced the expression of CYLD protein in J82/Dox and T24/Dox cells. ${ }^{*} p<0.05$ vs. blank control or miR-NC(+)

Firstly, the expression of CYLD was significantly down-regulated in doxorubicin-insensitive BUC tissues and cell lines compared with doxorubicin-sensitive BUC tissues and cell lines (Figures 3 B, C) and Spearman's correlation analysis identified a negative correlation between the expression of miR-130b and CYLD $(p<0.05)$.

Secondly, the luciferase reporter gene assay showed that miR-130b inhibited the relative luciferase activity by combining with the wild-type binding site, but not to the mutant binding site (Figure $3 \mathrm{D}$ ). Also, the miR-NC had no similar regulatory effects.

Thirdly, in J82/Dox and T24/Dox cells, western blot proved that miR-130b remarkably reduced the expression of CYLD protein (Figure $3 \mathrm{E}$ ).

\section{MiR-130b knockdown improved doxorubicin sensitivity of doxorubicin-resistant cells through negatively regulating CYLD}

Because miR-130b regulated doxorubicin sensitivity of J82/Dox and T24/Dox cells and the CYLD gene was a target of miR-130b, we conjectured that miR-130b modulated doxorubicin sensitivity of J82/Dox and T24/Dox cells through regulating CYLD. CCK8 assay revealed that the IC50 in the miR-130b(-) + sh-CYLD group to doxorubicin was higher compared with IC50 in the miR-130b(-) +

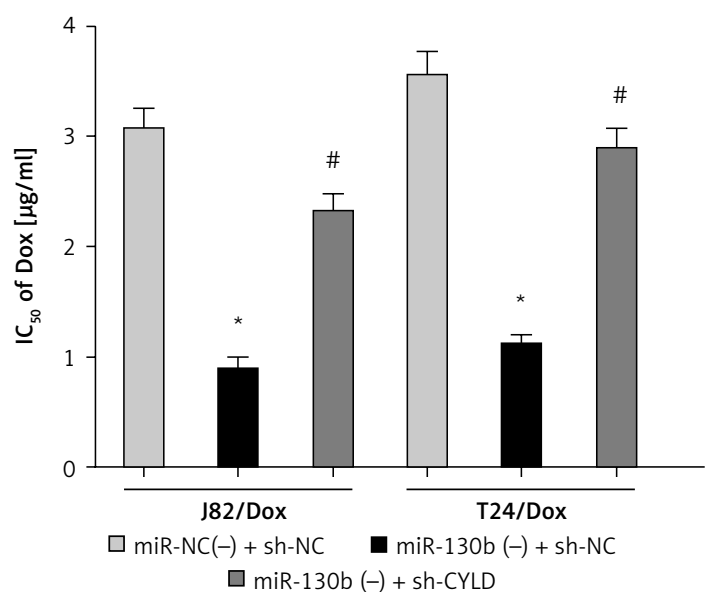

Figure 4. MiR-130b knockdown improved doxorubicin sensitivity of doxorubicin-resistant cells through negatively regulating CYLD. ${ }^{*} p<0.05$ vs. miR-NC(-) + sh-NC, ${ }^{\#} p<0.05$ vs. 130 b (-) + sh-NC 
sh-NC group, which revealed that miR-130b knockdown improved doxorubicin sensitivity of J82/Dox and T24/Dox cells through negatively regulating CYLD (Figure 4).

\section{Discussion}

It is well known that abnormal expression of microRNAs can regulate some pivotal biological behaviors, including chemotherapy resistance, which promoted chemotherapy failure and caused a poor prognosis [19-25]. This study firstly found that miR-130b was highly expressed in doxorubicin-insensitive BUC tissues and cell lines, which indicated that the aberrant expression of miR-130b was involved in chemotherapy resistance of BUC and its up-expression was negatively related to doxorubicin sensitivity in BUC. doxorubicin is a cytotoxic drug commonly used in systemic and intravesical chemotherapy in BUC patients, whose commodity name is doxorubicin. The following experiment found that knockdown of miR-130b reduced IC50 of J82/Dox and T24/Dox cells to doxorubicin. Yin et al. reported that CYLD could increase apoptosis and decrease autophagy to improve the chemosensitivity to gemcitabine in bladder cancer [26]. CYLD also was found to be involved in cisplatin (DDP) resistance in gastric cancer [26]. Therefore, miR-130b took part in the chemotherapy resistance of BUC, and miR-130b knockdown improved doxorubicin sensitivity of doxorubicin-resistant cells.

In consideration of the fact that microRNAs play their roles through regulating their target genes, for instance, miR-320a and STAT3, miR-210 and BNIP3 [27, 28], miR-130b might modify its target gene to regulate chemoresistance in doxorubicin-resistant cells in our study. Through the analysis of bioinformatics software TargetScan, the $3^{\prime}$-UTR of CYLD included a binding site of miR$130 \mathrm{~b}$, which revealed that the CYLD gene might be a target of miR-130b. Low CYLD expression has been detected in many kinds of cancer [14-16]. Some studies have reported that CYLD was a deubiquitinating enzyme and inhibited the activation of some signaling pathway, such as WNT/b-catenin and NF- $\mathrm{KB}[29,30]$. CYLD acted as a vital effector for biological behaviors, including migration, apoptosis and cell cycle [31-33].

Through a series of gain-of-function experiments, the CYLD gene was identified to be a target of miR-130b. Firstly, CYLD showed low expression in doxorubicin-insensitive BUC tissues and cell lines, and the expression of miR-130b showed a negative correlation with the CYLD expression. Secondly, luciferase reporter assay confirmed that miR-130b targeted combined with the 3'UTR of CYLD and inhibited the luciferase activity. Thirdly, the expression of CYLD protein could be silenced by over-expression of miR-130b.
Because miR-130b participated in doxorubicin resistance of BUC cells, and the CYLD gene is the target of miR-130b, miR-130b might modulate the doxorubicin sensitivity of BUC cells through regulating CYLD. To confirm the above speculation, sh-CYLD was used to silence the CYLD expression which was up-regulated by miR-130b knockdown in J82/Dox and T24/Dox cells, and the following experiments found that knockdown of CYLD reversed the regulatory roles of miR-130b knockdown in doxorubicin sensitivity of J82/Dox and T24/Dox cells. Accordingly, miR-130b knockdown improved doxorubicin sensitivity of doxorubicin-resistant cells through negatively regulating CYLD.

In conclusion, the over-expression of miR-130b is negatively related to doxorubicin sensitivity in BUC, and knockdown of miR-130b improves doxorubicin sensitivity in BUC by silencing CYLD expression. These findings might provide guidance for the clinical chemotherapy of BUC.

\section{Acknowledgments}

This work was supported by the National Nature Science Foundation of China (30901480, 81301834).

\section{Conflict of interest}

The authors declare no conflict of interest.

\section{References}

1. Yousef PG, Gabril MY. An update on the molecular pathology of urinary bladder tumors. Pathol Res Pract 2018; 214: 1-6.

2. Zheng HC. The molecular mechanisms of chemoresistance in cancers. Oncotarget 2017; 8: 59950-64.

3. Godbole M, Chandrani P, Gardi N, et al. miR-129-2 mediates down-regulation of progesterone receptor in response to progesterone in breast cancer cells. Cancer Biol Ther 2017; 18: 801-5.

4. Golbakhsh MR, Boddouhi B, Hatami N, et al. Down-regulation of microRNA-182 and microRNA-183 predicts progression of osteosarcoma. Arch Med Sci 2017; 13: 1352-6.

5. Li F, Liang A, Lv Y, Liu G, Jiang A, Liu P. MicroRNA-200c inhibits epithelial-mesenchymal transition by targeting the BMI-1 gene through the phospho-AKT pathway in endometrial carcinoma cells in vitro. Med Sci Monit 2017; 23: 5139-49.

6. Zhang HD, Jiang LH, Sun DW, Li J, Ji ZL. The role of miR130a in cancer. Breast Cancer 2017; 24: 521-7.

7. Li P, Wang X, Shan Q, Wu Y, Wang Z. MicroRNA-130b promotes cell migration and invasion by inhibiting peroxisome proliferator-activated receptor- $\gamma$ in human glioma. Oncol Lett 2017; 13: 2615-22.

8. Chang RM, Xu JF, Fang F, Yang H, Yang LY. MicroRNA-130b promotes proliferation and EMT-induced metastasis via PTEN/p-AKT/HIF-1 alpha signaling. Tumour Biol 2016; 37: 10609-19.

9. Egawa $H$, Jingushi $K$, Hirono $T$, et al. The miR-130 family promotes cell migration and invasion in bladder cancer 
through FAK and Akt phosphorylation by regulating PTEN. Sci Rep 2016; 6: 20574.

10. Miao Y, Zheng W, Li N, et al. MicroRNA-130b targets PTEN to mediate drug resistance and proliferation of breast cancer cells via the PI3K/Akt signaling pathway. Sci Rep 2017; 7: 41942.

11. Tian J, Hu L, Li X, Geng J, Dai M, Bai X. MicroRNA-130b promotes lung cancer progression via PPAR $\gamma /$ VEGF-A/ BCL-2-mediated suppression of apoptosis. J Exp Clin Cancer Res 2016; 35: 105.

12. Ratert N, Meyer HA, Jung M, et al. miRNA profiling identifies candidate miRNAs for bladder cancer diagnosis and clinical outcome. J Mol Diagn 2013; 15: 695-705.

13. Trompouki E, Hatzivassiliou E, Tsichritzis T, Farmer $\mathrm{H}$, Ashworth A, Mosialos G. CYLD is a deubiquitinating enzyme that negatively regulates NF-kappaB activation by TNFR family members. Nature 2003; 424: 793-6.

14. Lin X, Chen Q, Huang C, Xu X. CYLD promotes TNF-alpha-induced cell necrosis mediated by RIP-1 in human lung cancer cells. Mediators Inflamm 2016; 2016: 1542786.

15. Kotantaki P, Mosialos G. The expression of tumor suppressor gene Cyld is upregulated by histone deacetylace inhibitors in human hepatocellular carcinoma cell lines. Cell Biochem Funct 2016; 34: 465-8.

16. Hayashi M, Jono H, Shinriki S, et al. Clinical significance of cylindromatosis downregulation in breast cancer Breast Cancer Res Treat 2014; 143: 447-57.

17. Zhang H, Guo Y, Song Y, Shang C. Long noncoding RNA GAS5 inhibits malignant proliferation and chemotherapy resistance to doxorubicin in bladder transitional cell carcinoma. Cancer Chemother Pharmacol 2017; 79: 49-55.

18. Xie D, Zhang H, Hu X, Shang C. Knockdown of long non-coding RNA taurine up-regulated 1 inhibited doxorubicin resistance of bladder urothelial carcinoma via Wnt/ beta-catenin pathway. Oncotarget 2017; 8: 88689-96.

19. Hong Y, Shi Y, Shang C, Xue Y, Liu Y. Influence of far upstream element binding protein 1 gene on chemotherapy sensitivity in human U251 glioblastoma cells. Arch Med Sci 2016; 12: 156-62.

20. Zhao D, Zhang Y, Song L. MiR-16-1 targeted silences far upstream element binding protein 1 to advance the chemosensitivity to Adriamycin in gastric cancer. Pathol Oncol Res 2018; 24: 483-8.

21. Li S, Zhu J, Li J, Li S, Li B. MicroRNA-141 inhibits proliferation of gastric cardia adenocarcinoma by targeting MACC1. Arch Med Sci 2018; 14: 588-96.

22. Zhang H, Hu B, Wang Z, Zhang F, Wei H, Li L. miR-181C contributes to cisplatin resistance in non-small cell lung cancer cells by targeting Wnt inhibition factor 1 . Cancer Chemother Pharmacol 2017; 80: 973-84.

23. Xie D, Zhang H, Shang C. Long non-coding RNA CDKN2B antisense RNA 1 gene inhibits gemcitabine sensitivity in bladder urothelial carcinoma. J Cancer 2018; 9 . 2160-6.

24. Shang C, Tang W, Pan C, Hu X, Hong Y. Long non-coding RNA TUSC7 inhibits temozolomide resistance by targeting miR-10a in glioblastoma. Cancer Chemother Pharmacol 2018; 81: 671-8.

25. Yin L, Liu S, Li C, et al. CYLD downregulates livin and synergistically improves gemcitabine chemosensitivity and decreases migratory/invasive potential in bladder cancer: the effect is autophagy-associated. Tumour Biol 2016; 37: 12731-42.

26. Zhu M, Zhou X, Du Y, et al. miR-20a induces cisplatin resistance of a human gastric cancer cell line via targeting CYLD. Mol Med Rep 2016; 14: 1742-50.
27. Lv Q, Hu JX, Li YJ, et al. MiR-320a effectively suppresses lung adenocarcinoma cell proliferation and metastasis by regulating STAT3 signals. Cancer Biol Ther 2017; 18: 142-51.

28. Luan Y, Zhang X, Zhang Y, Dong Y. MicroRNA-210 protects PC-12 cells against hypoxia-induced injury by targeting BNIP3. Front Cell Neurosci 2017; 11: 285.

29. Pannem RR, Dorn C, Ahlqvist K, Bosserhoff AK, Hellerbrand C, Massoumi R. CYLD controls c-MYC expression through the JNK-dependent signaling pathway in hepatocellular carcinoma. Carcinogenesis 2014; 35: 461-8.

30. Mathis BJ, Lai Y, Qu C, Janicki JS, Cui T. CYLD-mediated signaling and diseases. Curr Drug Targets 2015; 16: 284-94.

31. Xia JT, Chen LZ, Jian WH, et al. MicroRNA-362 induces cell proliferation and apoptosis resistance in gastric cancer by activation of NF-kappaB signaling. J Transl Med 2014; 12: 33

32. Li D, Gao J, Yang Y, et al. CYLD coordinates with EB1 to regulate microtubule dynamics and cell migration. Cell Cycle 2014; 13: 974-83.

33. Wickström SA, Masoumi KC, Khochbin S, Fässler R, Massoumi R. CYLD negatively regulates cell-cycle progression by inactivating HDAC6 and increasing the levels of acetylated tubulin. EMBO J 2010; 29: 131-44. 